\title{
Less Iatrogenic Soft Tissue Damage Utilizing Robotic Assisted Total Knee Arthroplasty when Compared with a Manual Approach: A Blinded Assessment
}

\author{
Emily L. Hampp, $\mathrm{PhD}^{1}$; Nipun Sodhi, $\mathrm{BA}^{2}$; Laura Scholl, $\mathrm{PhD}^{1}$; Matthew E. \\ Deren, $\mathrm{MD}^{3}$; Zachary Yenna, $\mathrm{MD}^{3}$; Geoffrey Westrich, $\mathrm{MD}^{4}$; Michael A. \\ Mont, $\mathrm{MD}^{2}$ \\ ${ }^{1}$ Stryker, Mahwah, NJ USA \\ ${ }^{2}$ Department of Orthopaedic Surgery, Lenox Hill Hospital, Northwell Health, New York, NY \\ USA \\ ${ }^{3}$ Department of Orthopaedic Surgery, Cleveland Clinic, Cleveland, OH USA \\ ${ }^{4}$ Department of Orthopaedic Surgery, Hospital for Special Surgery, New York, NY USA \\ Emily.hampp@stryker.com
}

\begin{abstract}
While manual total knee arthroplasty (MTKA) has demonstrated excellent clinical results, occasionally intraoperative damage to soft tissues can occur. Robotic-arm assisted technology is designed to constrain a sawblade in a haptic zone to help ensure that only the desired bone cuts are made. The objective of this cadaver study was to quantify the extent of soft tissue damage sustained during TKA through a robotic-arm assisted (RATKA) haptically guided approach and conventional MTKA approach. Four surgeons each prepared 3 RATKA and 3 MTKA specimens for cruciate retaining TKAs. RATKA was performed on one knee, with MTKA on the other. Postoperatively, 2 additional blinded surgeons, assessed and graded damage to 14 key anatomic structures. A Kruskal-Wallis hypothesis test was performed to assess for statistical differences of soft tissue damages between RATKA and MTKA cases. A p-value $<0.05$ was used as the threshold for statistical significance, and p-values were adjusted for ties. Significantly less damage occurred to the PCL in the RATKA than the MTKA specimens $(\mathrm{p}<0.0001)$. RATKA specimens had less damage to the dMCL ( $\mathrm{p}=.149)$, ITB ( $\mathrm{p}=0.580)$, popliteus $(\mathrm{p}=0.248)$, and patellar ligament $(\mathrm{p}=0.317)$. The results of this study indicate that RATKA may result in less soft-tissue damage than MTKA, especially to the PCL.
\end{abstract}




\section{Introduction}

While manual total knee arthroplasty (MTKA) has demonstrated excellent clinical results, occasionally intraoperative damage to soft tissues can occur [1]. Soft tissue injury to the medial or lateral collateral ligaments (MCL or LCL), posterior cruciate ligament (PCL), or extensor mechanism may compromise post-operative clinical outcomes [2,3].

Stereotactically-guided bone preparation in robotic-arm assisted total knee arthroplasty (RA-TKA) is desired to constrain a sawblade in a haptic zone to help ensure that only desired bone cuts are made. The potential benefits to soft tissue protection have been clinically demonstrated for RA-TKA for posterior stabilized (PS) TKA, showing less iatrogenic soft tissue trauma than conventional TKA [4]. However, there is limited data characterizing these injuries, if any, for cruciate retaining (CR) TKA with the use of this technique. Therefore, the objective of this cadaver study was to compare the extent of soft tissue damage sustained during CR TKA through a robotic-assisted haptically-guided versus a conventional, manual TKA approach.

\section{Methods}

Twelve fresh-frozen pelvis-to-toe cadaver specimens ( 24 knees) were included: 6 females and 6 males. The mean age was 80.5 years (range $68-89$ ) and mean BMI was $26 \mathrm{~kg} / \mathrm{m}^{2}$ (range $21-36 \mathrm{~kg} / \mathrm{m}^{2}$ ). There was no sign of previous joint replacement or fracture. Four surgeons each prepared 3 RATKA and 3 MTKA specimens for cruciate retaining TKAs. RATKA was performed on one knee, with MTKA on the other. Postoperatively, 2 additional blinded surgeons, assessed and graded damage to 14 key anatomic structures according to the extent of percent damage (tissue fibers that were visibly torn, cut, frayed, or macerated over the total cross-sectional area) as defined in Table 1. The structures included: 1) Deep medial collateral ligament (dMCL);2) Superficial medial collateral ligament (sMCL); 3) Posterior oblique ligament; 4) Semimembranosus muscle tendon; 5) Gastrocnemius muscle medial head; 6) PCL; 7) Iliotibial band (ITB); 8) Lateral retinaculum; 9) LCL; 10) Popliteus tendon; 11) Gastrocnemius muscle lateral head; 12) Patellar ligament; 13) Quadriceps tendon; and 14) Extensor mechanism. A Kruskal-Wallis hypothesis test was performed to assess for statistical differences of soft tissue damages between RATKA and MTKA cases. A p-value $<0.05$ was used as the threshold for statistical significance, and p-values were adjusted for ties.

Table 1: Tissue damage scale

\begin{tabular}{|c|c|c|c|c|}
\hline Grade & $\mathbf{1}$ & $\mathbf{2}$ & $\mathbf{3}$ & $\mathbf{4}$ \\
\hline Damage & $\leq 5 \%$ & $6-25 \%$ & $26-75 \%$ & $76-100 \%$ \\
\hline
\end{tabular}

\section{Results}

Significantly less damage occurred to the PCL in the RATKA than the MTKA specimens $(\mathrm{p}<0.0001)$. RATKA specimens also had less damage to the dMCL $(\mathrm{p}=0.149)$, ITB $(\mathrm{p}=0.580)$, popliteus $(\mathrm{p}=0.248)$, and patellar ligament $(\mathrm{p}=0.317)$. The $\mathrm{sMCL}$, posterior oblique ligament, semimembranosus muscle tendon, gastrocnemius muscle medial head, gastrocnemius muscle lateral head, lateral retinaculum, LCL, quadriceps tendon, and extensor mechanism were grade 1 in all MTKA and RATKA specimens (Figures 1 and 2). No intentional soft tissue releases were performed in either group to balance the knee. 


\section{Conclusion}

This study was conceived to compare the amount of soft tissue damage found after performing TKAs with RATKA versus MTKA techniques. Our results show that less soft-tissue damage may occur utilizing the RATKA, especially for the PCL. These findings can potentially be attributed to the RATKA using a haptic boundary to constrain the sawblade, which can help prevent unwanted softtissue damage. The reduced soft tissue injury may also partly be due to tendency to create a full transverse tibial resection in the MTKA [4-6], whereas, the RATKA CR procedure is designed to leave a posterior bone island to help protect the PCL [7]. However, since any damage was post-operatively assessed and in a cadaveric model, further investigations on soft-tissue damage from patients with clinical outcomes should be performed. Nevertheless, this study provides a basis from which future clinical studies can be performed.

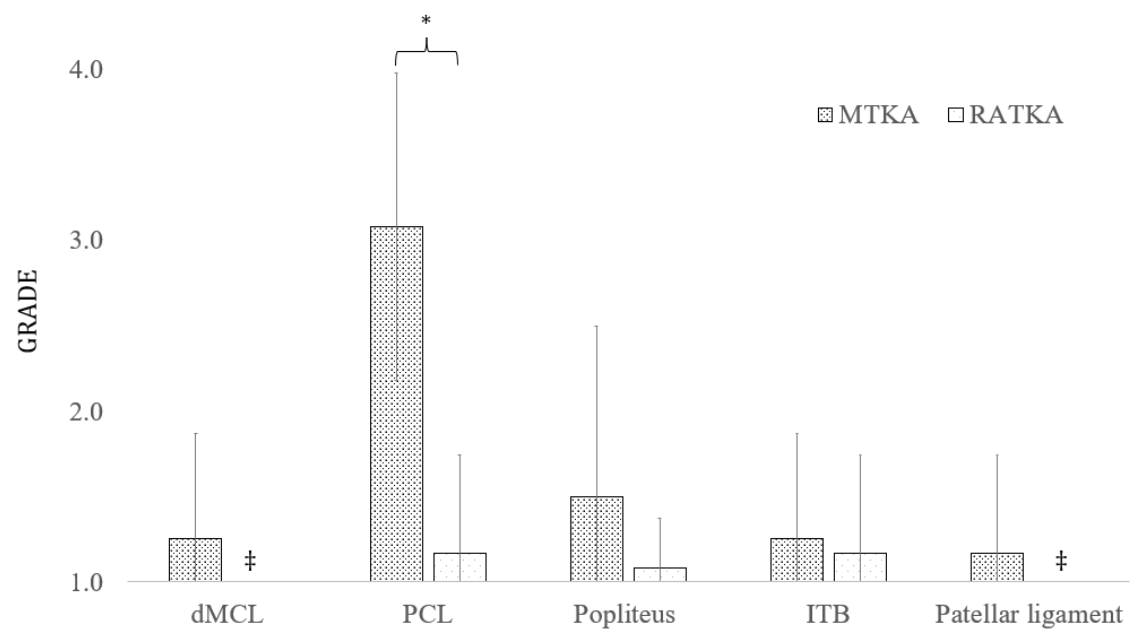

Figure 1. Bar chart showing the mean Grade 1 to 4 damage for the dMCL, PCL, popliteus, ITB, and patellar ligament in MTKA and RATKA specimens. Error bars indicate standard deviations. *PCL showed significant difference $(\mathrm{p}<0.05)$. †Grade mean \pm standard deviation for dMCL and patellar ligament was $1 \pm 0$.
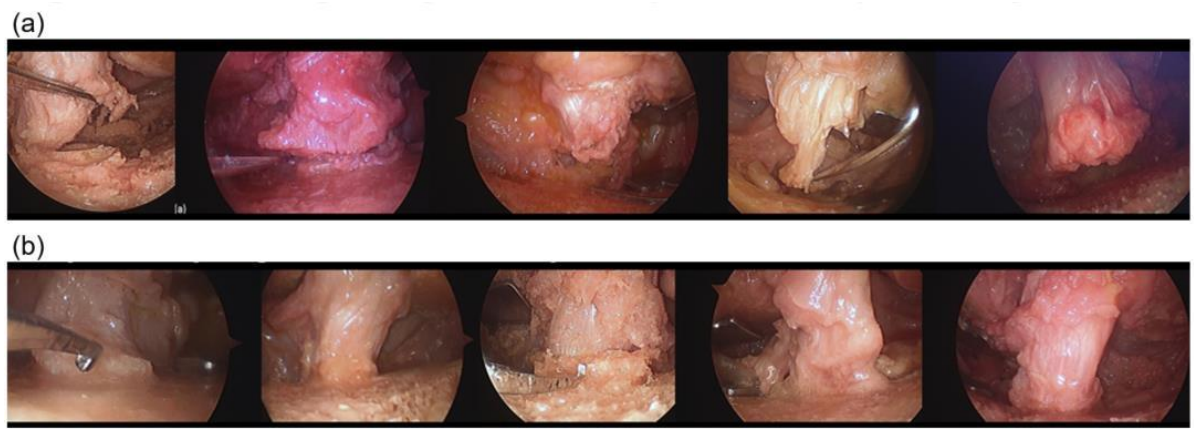

Figure 2: Examples of arthroscopic images of a) a severed PCL and b) an intact PCL, from MTKA and RATKA specimens, respectively. 


\section{References}

[1] Wijdicks CA, Griffith CJ, Johansen S, et al. Injuries to the medial collateral ligament and associated medial structures of the knee. J Bone Joint Surg Am 2010;92:1266-80.

[2] Kayani B, Konan S, Pietrzak JRT, Haddad FS. Iatrogenic Bone and Soft Tissue Trauma in RoboticArm Assisted Total Knee Arthroplasty Compared With Conventional Jig-Based Total Knee Arthroplasty: A Prospective Cohort Study and Validation of a New Classification System. J Arthroplasty. 2018 Aug;33(8):2496-2501. doi: 10.1016/j.arth.2018.03.042. Epub 2018 Mar 27.

[3] Bates MD, Springer BD. Extensor mechanism disruption after total knee arthroplasty. J Am Acad Orthop Surg 2015;23:95e106.

[4] Totlis T, Iosifidis M, Melas I, Apostolidis K, Agapidis A, Eftychiakos N, et al. Cruciate-retaining total knee arthroplasty: How much of the PCL is really retained? Knee Surgery, Sport Traumatol Arthrosc 2017;25:3556-60.

[5] Feyen H, Van Opstal N, Bellemans J. Partial resection of the PCL insertion site during tibial preparation in cruciate-retaining TKA. Knee Surgery, Sport Traumatol Arthrosc 2013;21:2674-9.

[6] Shannon FJ, Cronin JJ, Cleary MS, Eustace SJ, O'Byrne JM. The posterior cruciate ligamentpreserving total knee replacement: do we "preserve" it? J Bone Joint Surg Br 2007;89-B:766-71.

[7] Khlopas A, Chughtai M, Hampp EL, Scholl LY, Prieto M, Chang T-C, et al. Robotic-Arm Assisted Total Knee Arthroplasty Demonstrated Soft Tissue Protection. Surg Technol Int United States, 2017;30:441-446. 